Revista Médica Sinergia

Vol. 6, Núm. 8, agosto 2021, e753

\title{
Actualización en trastornos de conducta alimentaria: anorexia y bulimia nerviosa
}

Update on eating disorders: anorexia and bulimia nervosa

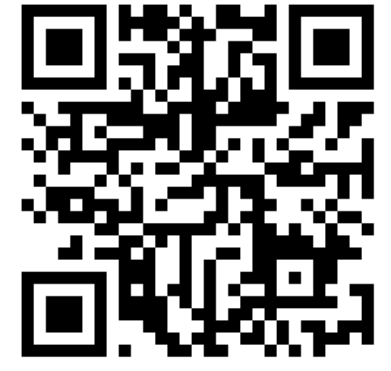

Recibido 05/05/2021
1Dra. Laura Vanessa Bermúdez Durán Investigadora independiente, Limón, Costa Rica https://orcid.org/0000-0002-4392-4794

${ }^{2}$ Dr. Mario Andrés Chacón Segura Investigador independiente, Alajuela, Costa Rica

(1) https://orcid.org/0000-0003-0859-2517

${ }^{3}$ Dra. Diana Melissa Rojas Sancho Investigadora independiente, Heredia, Costa Rica

(iD https://orcid.org/0000-0001-8339-3134

Corregido $15 / 05 / 2021$
Aceptado

20/06/2021

\section{RESUMEN}

Los trastornos de la conducta alimentaria son trastornos de la salud mental caracterizados por un comportamiento patológico frente a la ingesta alimentaria y una obsesión por el control de peso, acompañado de distorsión de la percepción de la imagen corporal. Las dos variantes más conocidas son la anorexia y la bulimia nerviosas. Presentan una etiología multifactorial, con una combinación de predisposición genética, influencias ambientales y rasgos psicológicos. Según el Manual Diagnóstico y Estadístico de los Trastornos Mentales en su quinta edición, el diagnóstico es clínico y debe ser temprano para tener un pronóstico más favorable. El rol de los psicofármacos en el manejo de estos trastornos es limitado, pero en varios estudios se muestra que la combinación de terapia cognitivo-conductual y farmacológica es el tratamiento más efectivo.

PALABRAS CLAVE: anorexia nerviosa; bulimia; psicoterapia; trastorno mental.

\section{ABSTRACT}

Eating disorders are mental health disorders characterized by pathological behavior towards food intake and an obsession with weight control, accompanied by distortion of the perception of body image. The two best known variants are anorexia nervosa and bulimia nervosa. They present a multifactorial etiology, with a combination of genetic predisposition, environmental influences, and psychological traits. According to the Diagnostic and Statistical Manual of Mental Disorders in its fifth edition, the diagnosis is clinical and must be early to have a more favorable prognosis. The role of psychotropic drugs in the management of these disorders is 
limited, but several studies have shown that the combination of cognitive-behavioral and pharmacological therapy is the most effective treatment.

KEYWORDS: anorexia nervosa; bulimia; psychotherapy; mental disorder.

${ }^{1}$ Médica general, graduada de la Universidad de Ciencias Médicas (UCIMED). Cód. MED16050. Correo: bermudezlau96@outlook.es

${ }^{2}$ Médico general, graduado de la Universidad de Ciencias Médicas (UCIMED). Cód. MED16091. Correo: marioa0894@gmail.com

${ }^{3}$ Médica general, graduada de la Universidad de Ciencias Médicas (UCIMED). Cód. MED16075. Correo: dianarojas2495@gmail.com

\section{INTRODUCCIÓN}

Los trastornos de conducta alimentaria (TCA) constituyen un grupo de trastornos mentales caracterizados por una conducta alterada ante la ingesta alimentaria y/o la aparición de comportamientos encaminados a controlar el peso (1). Se caracterizan por la insatisfacción de la percepción de la imagen corporal, miedo a engordar y pensamiento obsesivo con la alimentación (2). Las 2 variantes más conocidas son la anorexia nerviosa (AN) y la bulimia nerviosa (BN). Con frecuencia coexisten con otras enfermedades como la depresión, el abuso de sustancias o los trastornos de ansiedad (3). Estos trastornos tienen un origen multifactorial, dado que surgen de la interacción de factores psicológicos, físicos, sociales y culturales (4). La AN y BN muestran una gran variabilidad en su presentación y en su gravedad, lo que va a condicionar diferentes consideraciones terapéuticas y la individualización del tratamiento $(5,6)$.

En la actualidad, se conoce que la $A N$ o BN son condiciones médicas que alteran los procesos emocionales, cognitivos, asociados a un deterioro funcional que afecta la calidad de vida del individuo o incluso la vida en sí (1). El objetivo de esta revisión bibliográfica es brindar información relevante y actual a los profesionales en el área de la salud con respecto a la etiopatogenia de la enfermedad, conductas diagnósticas y terapéuticas a seguir ante la sospecha y confirmación de un paciente con AN o BN, y mejorar la calidad de vida de estas personas.

\section{MÉTODO}

Para la elaboración de este articulo tipo revisión bibliográfica, se revisaron publicaciones con fechas de 5 años previos en bases de datos como Pubmed, Medline, Asociación Americana de Psiquiatría, British Journal of Psichiatry, Journal of the American Medical Association (JAMA) y buscadores como Google Académico. Dentro de los resultados se revisaron los artículos y se eligieron los que presentaron mayor respaldo y relevancia en la comunidad científica. Se utilizaron un total de 17 referencias bibliográficas.

\section{ANOREXIA NERVIOSA}

El término AN deriva del término griego para «pérdida de apetito» y una palabra latina que implica un origen nervioso. Es un síndrome caracterizado por tres criterios fundamentales: una búsqueda implacable de la delgadez o un miedo mórbido a la obesidad, es decir, una psicopatología, una inanición autoinducida de una intensidad significativa, esto es, un comportamiento, y la presencia de signos y síntomas médicos debidos a la inanición, esto es síntomas fisiológicos $(1,7)$.

\section{EPIDEMIOLOGÍA}

La AN es la enfermedad psiquiátrica más frecuente en las mujeres jóvenes y la tercera 
enfermedad crónica tras el asma y la obesidad en las adolescentes (5), además los TCA se consideran el principal problema psiquiátrico de la pubertad y adolescencia en ambos sexos (6). Las edades de inicio más habituales se sitúan en la mitad de la adolescencia, entre los 14 y los 18 años, pero hasta en el $5 \%$ de los pacientes se inicia a principios de la década de los 20 años. Se estima que la prevalencia de la AN se da en alrededor del $0,5-1 \%$ de las adolescentes. Es de 10 a 20 veces más frecuente en las mujeres que en los hombres (1). Los cambios en los criterios diagnósticos introducidos por el DSM-5 se tradujeron en un incremento de casos catalogados como AN y BN, y una reducción significativa de aquellos identificados como TCA no especificados $(8,9)$.

\section{ETIOLOGÍA}

Existen múltiples causas de los $\mathrm{AN}$; esta patología surge de la interacción de factores psicológicos, biológicos, sociales y culturales que impactan en el comportamiento del individuo (4). La preocupación excesiva por el peso o los antecedentes de dieta parecen indicar un mayor riesgo, se identificó una predisposición genética sin haberse identificado aún las variantes genéticas asociadas, además la desregulación en los sistemas dopaminérgico y serotoninérgico, más las alteraciones en los circuitos neurales relacionados con la recompensa y el autocontrol son ejemplos de factores neurobiológicos relacionados con estas enfermedades $(8,9)$.

En la sociedad occidental, la obesidad se considera poco atractiva y saludable; el deseo de ser delgado es dominante, incluso entre los niños. Más del $50 \%$ de las niñas en edad prepuberal hacen dieta o adoptan otras medidas para controlar su peso. Algunos antecedentes comunes en la $\mathrm{AN}$ son la preocupación excesiva por el peso, la obsesión por un cuerpo delgado, el distrés sociofamiliar, el antecedente de realización de una dieta y el de abuso sexual en la infancia $(10,11)$.

\section{DIAGNÓSTICO Y CUADRO CLÍNICO}

El diagnóstico de la AN es fundamentalmente clínico y se basa en los tres criterios del Según el Manual Diagnóstico y Estadístico de los Trastornos Mentales, quinta edición (DMS-5) (12).

- Criterio A. Restricción de la ingesta energética en relación con las necesidades, que conduce a un peso corporal significativamente bajo con relación a la edad, el sexo, el curso de desarrollo y la salud física. Peso significativamente bajo se define como un peso que es inferior al mínimo normal 0 , en niños y adolescentes inferior a mínimo esperado.

- Criterio B. Miedo intenso a ganar peso o a engordar, o comportamiento persistente que interfiere en el aumento de peso, incluso con un peso significativamente bajo.

- Criterio C. Alteración en la forma en que uno mismo percibe su propio peso o constitución, influencia impropia del peso o la constitución corporal en a autoevaluación, o falta persistente de reconocimiento de la gravedad del bajo peso corporal actúa.

Todos los pacientes con AN tienen un gran miedo a aumentar de peso y convertirse en obesos, lo que indudablemente contribuye a su falta de interés, o incluso a la resistencia al tratamiento. Presentan conductas restrictivas, como reducción de las porciones de comida ingerida, ayunos, evitación de cierto tipo de alimentos, presencia de reglas estrictas relacionadas con la alimentación, conductas ritualizadas en la compra, preparación y consumo de alimentos, y evitación de las comidas habituales en el 
hogar y del comer en situaciones sociales $(8,9)$. La conducta obsesivo-compulsiva, la depresión y la ansiedad son síntomas psiquiátricos de la AN destacados con frecuencia en el cuadro clínico. La mayoría de las conductas anormales para perder peso se producen en secreto (1). Además, los pacientes tienen alteración en la imagen corporal, con miedo excesivo de ganar peso (6).

La AN se ha dividido en dos subtipos: el tipo restrictivo y el tipo con atracones/purgas. En el tipo restrictivo, presente en alrededor del $50 \%$ de los casos, la ingesta de alimentos está muy restringida, se describe presentaciones en las que la pérdida de peso es debida sobre todo a la dieta, el ayuno y/o ejercicio excesivo (1). En el tipo con atracones/purgas, los pacientes van alternando los intentos de seguir una dieta rigurosa con episodios intermitentes de atracones o purgas (es decir, vómitos autoprovocados o utilización incorrecta de laxantes, diuréticos o enemas). A veces se dan purgas repetidas sin que antes se hayan producido atracones, tras haber ingerido solo unas pocas calorías $(8,12)$.

\section{EXPLORACIÓN FÍSICA Y PRUEBAS ANALÍTICAS}

En los pacientes con AN se observa al examen físico rápida pérdida de peso a lo largo de varias semanas o meses, tienden a usar ropa suelta, presentan piel seca, hipercarotinemia, edemas por hipoproteinemia, lanugo, estreñimiento, distención abdominal y enlentecimiento del vaciado gástrico, hipotermia e intolerancia al frio, debilidad y laxitud general. EI ECG puede mostrar prolongación Q-T (signo de riesgo) cambios en el segmento ST y en la onda $\mathrm{T}$, que suelen ser secundarios a las alteraciones electrolíticas; además presentan hipotensión y bradicardia $(1,6)$. El hemograma completo revela con frecuencia anemia y una leucocitopenia con linfocitosis relativa. $\mathrm{Si}$ se producen atracones y purgas, la determinación de electrólitos séricos muestra una alcalosis hipopotasémica (1). Las concentraciones de cloro y potasio son bajas, en cambio la amilasa es alta si el paciente tiene vómitos. Los pacientes que usan laxantes tienden a presentar una acidosis metabólica por perdida en las diarreas (8). Los cambios endocrinos que se producen, como la amenorrea, el hipotiroidismo leve y la hipersecreción de la hormona liberadora de corticotropina, están causados por la situación de bajo peso y vuelven a la normalidad con el aumento de peso $(1,9)$.

La AN se asocia a numerosas complicaciones médicas, que se relacionan con la intensidad del trastorno, la duración del mismo y el predominio del patrón alimentario restrictivo, compulsivo $\mathrm{y} / \mathrm{o}$ purgativo (11,13). Las complicaciones médicas de la AN generalmente se deben a la desnutrición y la pérdida de peso y sus compensaciones fisiológicas asociadas (14).

\section{COMORBILIDAD}

En general, esta se asocia a depresión en el $65 \%$ de los casos, a fobia social en el $34 \%$, y al trastorno obsesivo-compulsivo en el $26 \%$ (1). Otros padecimientos psiquiátricos asociados son el trastorno obsesivo compulsivo, ataques de pánico, déficit de atención e hiperactividad, desorden bipolar, abuso de sustancias y síndrome de estrés postraumático (6).

\section{DIAGNOSTICO DIFERENCIAL}

Se deben descartar enfermedades orgánicas que puedan explicar la pérdida de peso, como en muchos tipos de cáncer, diabetes mellitus, hipertiroidismo, colitis ulcerosa, celiaquía, entre otros (15). Por otra parte, diferentes trastornos psiquiátricos pueden cursar con pérdida de peso, rumiaciones obsesivas, episodios de llanto y 
falta de apetito, como a veces ocurre en la depresión, pero en esta enfermedad no existe el miedo a engordar típico de la AN. Comparada con la agitación nerviosa, la hiperactividad que se observa en la an está planificada y ritualizada. Las fluctuaciones del peso, los vómitos y las peculiaridades en el manejo de la comida pueden darse en el trastorno de somatización $(1,11)$.

Pueden aparecer conductas inusuales en la esquizofrenia o conductas de evitación de comer en público en la fobia social. Es más probable un paciente esquizofrénico crea que la comida está envenenada. Estos pacientes casi nunca están preocupados por convertirse en obesos y no muestran la hiperactividad que se observa en la $A N$. Además, es de suma importancia diferenciar de otros trastornos de conducta alimentaria $(10,4)$.

\section{EVOLUCIÓN}

La evolución de la AN considerada en su globalidad es muy variable: recuperación espontánea sin tratamiento, recuperación tras una serie de tratamientos, evolución fluctuante de aumentos de peso seguidos de recaídas, y evolución gradualmente degenerativa que da lugar a la muerte debido a las complicaciones de la desnutrición $(1,15)$.

\section{PRONÓSTICO}

En general, el pronóstico no es bueno. Los estudios han descrito unos índices de mortalidad de aproximadamente $6 \%$, siendo fundamentalmente como consecuencia de la inanición o por suicidios. Si bien el pronóstico es variable de acuerdo con el momento del diagnóstico y el tipo de padecimiento, existen algunos factores asociados con mal pronóstico, tales como las conductas purgativas, neuroticismo infantil, la BN, trastornos de personalidad, conflictos familiares y edad de presentación temprana o tardía $(1,6)$.

Los indicadores de un desenlace favorable son el reconocimiento de la sensación de hambre, la reducción de la negación y de la inmadurez, así como la mejora de la autoestima (1). Tanto el reconocimiento temprano, como el manejo adecuado de los TCA resultan cruciales para un pronóstico favorable (9).

\section{TRATAMIENTO}

El tratamiento de los pacientes de la AN puede realizarse en distintos niveles asistenciales y debe ser siempre multidisciplinar, siendo los pilares fundamentales la psicoterapia, tratamiento farmacológico y el abordaje nutricional $(1,5)$.

- Psicoterapia: la terapia psicológica que se recomienda es la terapia cognitivoconductual (TCC) (1). Esta terapia está destinada a apoyar la adherencia al tratamiento multidisciplinario $\mathrm{e}$ indicaciones nutricionales; aumentar la motivación a mantener un peso saludable y cesar las conductas propias del TCA; desarrollar una comprensión compartida de los problemas que subyacen al TCA; facilitar que el paciente retome as actividades diarias normales; y prevenir recaídas $(8,9)$. La psicoterapia dinámica expresiva de apoyo y famiiar se usa a veces en el tratamiento de pacientes con AN, pero la resistencia de estos puede convertirla en un proceso difícil y doloroso (1).

- Manejo nutricional: el manejo nutricional está dirigido a la corrección de las alteraciones nutricionales, la conducta alimentaria y otros comportamientos destinados a un control ponderal no saludable, además de al establecimiento de un manejo saludable y flexible de la alimentación y el peso (8). La educación nutricional debe formar parte del tratamiento de la 
AN y estar orientada a la acción y centrada en la práctica. Su objetivo principal es facilitar la adopción voluntaria de comportamientos alimentarios que fomenten la salud y el bienestar. Debe plantearse no solo de acuerdo al diagnóstico del trastorno, sino también debe tener en cuenta el patrón alimentario del paciente y sus conocimientos nutricionales $(5,6)$.

- Farmacología: el rol de los psicofármacos en el manejo de los TCA es limitado, con escasa evidencia, debiendo utilizarse principalmente para el tratamiento de la psicopatología comórbida. En AN, se ha sugerido el uso de antipsicóticos con efectos sedativos, tales como la olanzapina, en casos en que el paciente se encuentra extremadamente agitado y con resistencia a la realimentación (8). Algunas publicaciones respaldan el empleo de la ciproheptadina, un fármaco con propiedades antihistamínicas y antiserotoninérgicas, en pacientes con anorexia nerviosa del tipo restrictivo (9). También se han descrito algunos beneficios con amitriptilina, pero los estudios farmacológicos todavía no han identificado un fármaco que proporcione una mejora definitiva de los síntomas esenciales de la anorexia nerviosa (1).

Está indicado el ingreso hospitalario a los pacientes cuyo peso esté un 25 a $30 \%$ por debajo del recomendado para su altura, si presentan trastornos psiquiátricos graves, grave conflicto familiar generado por el trastorno alimentario y/o fracaso confirmado y reiterado del tratamiento ambulatorio o del hospital de día (1).

\section{BULIMIA NERVIOSA}

La BN es un TCA en el que una persona presenta episodios de ingesta exagerada de alimentos en periodos cortos de tiempo, acompañadas de conductas compensatorias inapropiadas de manera repetida que eviten el aumento de peso por los atracones (7). El malestar físico (p. ej., dolor abdominal o náuseas) dan fin al atracón, que a menudo va seguido por sentimientos de culpa, depresión o disgusto con uno mismo (10).

\section{EPIDEMIOLOGÍA}

La $B N$ es más prevalente que la $A N$. Las estimaciones de prevalencia oscilan entre un $1.2 \%$ y $4 \%$ en las mujeres jóvenes (9). Al igual que con la $\mathrm{AN}$, la $\mathrm{BN}$ es significativamente más frecuente en las mujeres que en los hombres, pero a menudo su inicio se produce en etapas más avanzadas de la adolescencia que en la AN (1). La edad promedio de inicio es entre los 16 y 20 años, pero puede producirse incluso durante las primeras fases de la vida adulta $(10,16)$.

\section{ETIOLOGÍA}

Se cree que la etiología de la $B N$, al igual que la AN, es multifactorial, con una combinación de predisposición genética, influencias ambientales y rasgos psicológicos que influyen (10). En la BN, la familia como primer agente de socialización de la persona, posibilita el acceso y adaptación de sus hijos a la sociedad. Es por eso que la familia adquiere un lugar prioritario para el desarrollo físico y psicológico de sus miembros (8). Socialmente, los pacientes con $\mathrm{BN}$ tienden a mostrar un alto rendimiento y a responder a las presiones de la sociedad a favor de la delgadez. Asimismo, muchos están deprimidos y tienen un mayor grado de depresión familiar, como sucede en los pacientes con AN, pero las familias de los primeros suelen ser menos cercanas y más conflictivas que las de estos últimos (1).

\section{DIAGNÓSTICO Y CUADRO CLÍNICO}


El diagnóstico de la $\mathrm{AN}$ es fundamentalmente clínico y se basa en los tres criterios del Según el DMS-5 (12).

- Criterio A. Episodios recurrentes de atracones, caracterizados por ambos:

1. Ingestión en un periodo determinado, de una cantidad de alimentos que es claramente superior a la que a mayoría de las personas ingerirían en un periodo similar en circunstancias parecidas.

2. Sensación de falta de control sobre lo que se ingiere durante el episodio.

- Criterio B. Comportamientos compensatorios inapropiados recurrentes para evitar el aumento de peso, como el vómito autoinducido, uso incorrecto de laxantes, diuréticos u otros medicamentos; ayuno 0 ejercicio excesivo.

- Criterio C. Los atracones y los comportamientos compensatorios inapropiados se producen, en promedio, al menos una vez a la semana durante 3 meses.

- Criterio D. La autoevaluación se ve indebidamente influida por la constitución y el peso del cuerpo.

- Criterio E. La alteración no se produce exclusivamente durante episodios de AN.

Las pacientes bulímicas comparten con las anoréxicas el rechazo a ganar peso y la preocupación centrada en aspectos y peso. Las pacientes con BN tienen mayor conciencia de la enfermedad, si bien niegan su trastorno en fases iniciales, es más fácil que reconozcan el trastorno y la gravedad de sus conductas respecto a las comidas, mejorando así su pronóstico $(1,4)$.

El atracón se caracteriza por la aparición de episodios recurrentes, de unas dos horas de duración, durante los cuales se ingiere grandes cantidades de alimentos, de forma rápida y acompañado de una sensación de pérdida de control sobre la ingesta (12).
Durante los atracones, los pacientes ingieren alimentos dulces, ricos en calorías $y$, por lo general, de textura blanda o lisa, como los pasteles y la bollería (1). Estos episodios se deben sospecharse en jóvenes que aumentan progresivamente de peso a pesar de evidenciar una ingesta disminuida o normal durante las comidas habituales (8). Posteriormente realizan conductas compensatorias, tanto purgativas como vómitos autoinducidos y uso indebido de laxantes, diuréticos y/u otros medicamentos o no purgativas tales como restricción alimentaria y/o ejercicio excesivo (9).

Los vómitos son comunes y suelen provocarse introduciendo un dedo en la garganta, aunque algunos pacientes son capaces de vomitar a voluntad. Estos reducen el dolor abdominal y la sensación de estar hinchado, y permiten que los pacientes sigan comiendo sin temor al aumento de peso (1). Con frecuencia aparece tras el episodio la depresión, denominada en ocasiones angustia post atracón. Las complicaciones de la $\mathrm{BN}$ se deben a la frecuencia y al modo de estos comportamientos (14). En las conductas purgativas tienden a esconderse (por culpa, vergüenza o para evitar suspenderlas) y pueden pasar desapercibidas durante años. Deben sospecharse, por ejemplo, cuando existen visitas frecuentes al baño durante y después de las comidas (9). La mayoría de los pacientes con BN se encuentran en un rango de peso normal, pero algunos pueden estar por debajo de este o tener sobrepeso, además no suele haber amenorrea. Estos pacientes están preocupados por su silueta corporal y por su aspecto, se preocupan por cómo les ven los demás y por su atractivo sexual (1).

\section{EXPLORACIÓN FÍSICA Y PRUEBAS ANALÍTICAS}

En general, en la BN se conserva intacta la función del tiroides, pero los pacientes 
pueden mostrar una falta de supresión en la prueba de supresión con dexametasona. Es probable que los pacientes que recurren regularmente a las purgas muestren deshidratación y desequilibrios electrolíticos tales como hipopotasemia, hiponatremia, hipomagnesemia, hipocloremia y alcalosis metabólica (vómitos), acidosis metabólica (laxantes) y hiperamilasemia $(1,8)$. En el examen físico es muy característico la callosidad en el dorso de a mano conocido como signo de Russell, además el ácido del vómito puede dañar el esmalte dental, un hallazgo habitual en estos pacientes (4).

\section{COMORBILIDAD}

Hay mayor comorbilidad con trastornos afectivos, abuso de sustancias y trastornos de personalidad que en la AN (1).

\section{DIAGNOSTICO DIFERENCIAL}

A los individuos con atracones que aparecen exclusivamente en el transcurso de la $A N$ se les diagnostica AN, tipo compulsivo/purgativo, y no debe establecerse el diagnóstico adicional de BN $(3,8)$. Los médicos deben confirmar que los pacientes no tengan enfermedades neurológicas o médicas, como convulsiones de tipo epiléptico, tumores del sistema nervioso central, síndrome de Klüver - Bucy o síndrome de Kleine - Levin donde se ha observado una conducta alimentaria alterada (1). El comer en exceso es frecuente en el trastorno depresivo mayor, con síntomas atípicos, pero estos sujetos no recurren a medidas compensatorias ni muestran preocupación excesiva por el peso y la silueta corporales (3).

\section{EVOLUCIÓN}

La BN se caracteriza por tasas más elevadas de recuperación parcial y completa que la AN. Los pacientes tratados tienen una evolución mucho mejor que los que no reciben tratamiento (1). Estos pacientes tienen a la cronicidad y es fluctuante con periodos de normalidad y recaídas frecuentes. Los no tratados tienden a la cronicidad o pueden mostrar pequeños grados de mejoría, en general poco destacables, con el tiempo (6).

\section{PRONÓSTICO}

En el DSM-5, la mortalidad por BN se ha estimado en un $2 \%$ por década (12). Si bien el pronóstico es variable de acuerdo con el momento del diagnóstico y el tipo de padecimiento, existen algunos factores asociados con mal pronóstico, tales como las conductas purgativas, antecedente de consumo de sustancias, trastornos de personalidad, conflictos familiares y la mayor duración del trastorno en el momento de la presentación. Son factores de buen pronóstico el inicio en edades tempranas y el tener poco tiempo de evolución $(1,6)$.

\section{TRATAMIENTO}

La mayor parte de los pacientes con BN no complicada no necesitan ser ingresados. En general, no son tan reservados con respecto a sus síntomas como los pacientes con AN, por lo que el tratamiento ambulatorio no suele ser complicado, aunque a menudo la psicoterapia es tormentosa y puede ser prolongada (1).

La mayor parte de los pacientes con BN no complicada no necesitan ser ingresados (1). En general, no son tan reservados con respecto a sus síntomas, por lo que el tratamiento ambulatorio no suele ser complicado, aunque a menudo la psicoterapia es tormentosa y puede ser prolongada. La combinación de psicoterapia con antidepresivos puede ser el tratamiento más eficaz para superar este trastorno $(10,8)$. 
- Psicoterapia: La TCC se considera el tratamiento de elección, además de tener mejor respuesta que en los pacientes con AN. La TCC implementa una serie de intervenciones cognitivas y conductuales para: 1) interrumpir el ciclo conductual automantenido de atracones y realización de dietas, y 2) modificar los pensamientos disfuncionales del individuo: creencias en torno a la comida, el peso, la imagen corporal y el autoconcepto general $(1,17)$.

- Tratamiento farmacológico: Se ha demostrado que los antidepresivos son útiles para tratar la BN. Estos incluyen los inhibidores selectivos de la recaptación de serotonina como la fluoxetina (1). En adolescentes con BN, un estudio clínico demostró que la fluoxetina en dosis usadas en adultos ( 60 $\mathrm{mg} /$ día) añadida a la psicoterapia de apoyo, disminuyó significativamente los atracones y purgas, sin embargo, la evidencia es aún limitada $(17,8)$. La imipramina, la desipramina, la trazodona y los inhibidores de la monoaminooxidasa han resultado útiles (1).

Puede ser necesaria la hospitalización por deshidratación, hipopotasemia, arritmia, inestabilidad médica (bradicardia grave, hipotensión ortostática y convulsiones), atracones y purgas incontrolables, tendencias suicidas 0 fracaso del tratamiento ambulatorio (10).

\section{CONCLUSIONES}

La AN y BN son enfermedades graves, crónicas y multifactoriales. El conocimiento de su etiología, clínica, comorbilidades y complicaciones nos permite un enfoque integral de tratamiento, en donde la educación de los familiares es fundamental para el manejo y seguimiento. Los criterios diagnósticos y el examen físico permiten al médico de primer contacto, la detección y el inicio de terapia temprana, que llevan a un mejor pronóstico.

Es de suma importancia que el personal médico tenga el conocimiento general de las características clínicas y el adecuado manejo de a $\mathrm{AN}$ y BN, además de recalcar el énfasis en la salud mental y sus factores de riesgo, para así poder realizar estrategias más optimas por parte del todo el personal de salud y así un manejo y seguimiento multidisciplinario más adecuado para cada paciente.

\section{Los autores declaran no tener conflicto de interés.}

\section{REFERENCIAS}

1. Sadock, B., Sadock, V., Kaplan, H. and Ruiz, P., 2015. Sinopsis De Psiquiatría. 11th ed. Barcelona: Wolters Kluwer, pp.962-980.

2. Ponce Torres C, Turpo Espinoza K, Salazar Pérez C, Viteri-Condori L, Carhuancho Aguilar J, Taype Rondan Á. Trastornos de la conducta alimentaria en estudiantes de medicina de una universidad de Perú. Rev Cub Salud Publica. 2017;43:551-61. Disponible

en: https://www.scielosp.org/article/rcsp/2017.v43n4/ 551-561/es/

3. Treasure J, Duarte TA, Schmidt U. Eating disorders. Lancet. 2020;395(10227):899-911. Disponible en: https://pubmed.ncbi.nlm.nih.gov/32171414/

4. Universidad Autónoma del Estado de Hidalgo. Vista de Trastornos de la Conducta Alimentaria [Internet]. Edu.mx. [cited 2021 May 5]. Available from:

https://repository.uaeh.edu.mx/revistas/index.php /atotonilco/article/view/6036/7243

5. Gómez-Candela C, Palma-Milla S, Miján-de-laTorre A, Rodríguez-Ortega $\mathrm{P}$, Matía-Martín $\mathrm{P}$, Loria-Kohen V, et al. Consenso sobre la evaluación y el tratamiento nutricional de los trastornos de la conducta alimentaria: anorexia nerviosa, bulimia nerviosa, trastorno por atracón y otros. Resumen ejecutivo. Nutr Hosp. 2018;35(2):489-94. Disponible en: http://scielo.isciii.es/scielo.php?pid=S021216112018000200489\&script=sci arttext\&tlng=en

6. Ayuzo-del Valle NC, Covarrubias-Esquer JD. Trastornos de la conducta alimentaria. Rev mex pediatr. 2019;86(2):80-6. Disponible en: 
http://www.scielo.org.mx/scielo.php?pid=S003500522019000200080\&script=sci arttext

7. Lladó G, González-Soltero R, Blanco Fernández de Valderrama MJ. Anorexia y bulimia nerviosas: difusión virtual de la enfermedad como estilo de vida. Nutr Hosp. 2017;34(3):693-701. Disponible en:

http://scielo.isciii.es/scielo.php?script=sci arttext \&pid=S0212-16112017000300693

8. Gaete P V, López C C. Eating disorders in adolescents. A comprehensive approach. Rev Chil Pediatr. 2020;91(5):784-93. Disponible en: https://scielo.conicyt.cl/scielo.php?pid=S037041062020000500784\&script=sci arttext

9. Losada AV, Lupis BL. Etiología y Prevención de Bulimia Nerviosa y Anorexia Nerviosa. Psicol para am lat www,psicolatina,org. 2018;(30):26-43.

10. Castillo $M$, Weiselberg $E$. Bulimia nervosa/purging disorder. Curr Probl Pediatr Adolesc Health Care. 2017;47(4):85-94. Disponible en: https://pubmed.ncbi.nlm.nih.gov/28532966/

11. Gómez-Candela C, Palma-Milla S, Miján-de-laTorre A, Rodríguez-Ortega P, Matía-Martín P, Loria-Kohen $\mathrm{V}$, et al. Consenso sobre la evaluación y el tratamiento nutricional de los trastornos de la conducta alimentaria: anorexia nerviosa, bulimia nerviosa, trastorno por atracón y otros. Resumen ejecutivo. Nutr Hosp. 2018;35(2):489-94. Disponible en: http://scielo.isciii.es/scielo.php?pid=S021216112018000200489\&script=sci arttext\&tIng=en

12. Asociación Americana de Psiquiatría, Guía de consulta de los criterios diagnósticos del DSM 5. Arlington, VA, Asociación Americana de Psiquiatría, 2013.

13. Gabler $G$, Olguín $P$, Rodríguez $A$. COMPLICACIONES MÉDICAS DE LOS TRASTORNOS DE LA CONDUCTA ALIMENTARIA. Rev médica Clín Las Condes. 2017;28(6):893-900.

14. Gibson D, Workman C, Mehler PS. Medical complications of anorexia nervosa and bulimia nervosa. Psychiatr Clin North Am. 2019;42(2):263-74.

15. Ortiz Cuquejo LM, Aguiar C, Samudio Domínguez GC, Troche Hermosilla A. Eating Disorders in adolescents: A booming disease? Pediatr (Asunción). 2017;44(1):37-42. Disponible en: https://www.revistaspp.org/index.php/pediatria/art icle/view/151/146

16. Santonicola A, Gagliardi M, Guarino MPL, Siniscalchi M, Ciacci C, lovino P. Eating disorders and gastrointestinal diseases. Nutrients [Internet]. 2019;11(12). Available from: http://dx.doi.org/10.3390/nu11123038

17. Gorrell S, Le Grange D. Update on treatments for adolescent bulimia nervosa. Child Adolesc Psychiatr Clin N Am. 2019;28(4):537-47.
Disponible https://pubmed.ncbi.nlm.nih.gov/31443872/ en: 